\title{
The Impact of Inhaled Corticosteroids Used for the Treatment of Asthma on the Management of Diabetes Mellitus
}

\author{
*Dasdemir Ilkhan Gulay ${ }^{1}$ Celikhisar Hakan ${ }^{2}$ \\ 1.Tire Public Hospital, Chest Diseases Clinic, Tire-Izmir-Turkey \\ 2.Esrefpasa Metropolitan Municipality Hospital, Chest Diseases Clinic, Izmir-Turkey
}

\begin{abstract}
In this study, it is aimed to investigate the impact of inhaled corticosteroids on the management of diabetes in diabetic patients diagnosed with asthma. This is a retrospective case control study. 188 patients diagnosed with diabetes mellitus and 88 diabetes mellitus patients diagnosed with asthma - 276 patients in total - were enrolled in the study. Patients using systemic steroids for any reason, patients diagnosed with diabetes, who do not take medication regularly, patients with diabetic ketoacidosis and patients, who had an asthma episode requiring the use of oral steroids in the past year were excluded from the study. A total of 276 patients; 88 (31.9\%) male and $188(68.1 \%)$ female were enrolled in the study with the average age being 58.9 $9.4 .68 .1 \%$ (188) of the patients enrolled in the study were diagnosed with diabetes mellitus and $31.9 \%$ (88) were diabetes mellitus patients diagnosed with asthma. The comparison between the group not diagnosed with asthma and the group diagnosed with asthma, blood glucose $(p<0.007)$ levels and incidence of neuropathy $(\mathrm{p}<0.002)$ were significantly higher in patients diagnosed with asthma and incidence of nephropathy $(\mathrm{p}<0.024)$ was significantly higher in patients not diagnosed with asthma. In our study, no statistically significant differences were observed between the two groups with regard to age, haemoglobin levels and glycated haemoglobin levels. In conclusion, the use of low-medium dose inhaled corticosteroids in diabetes mellitus patients diagnosed with asthma does not have an impact on glycated haemoglobin levels but with regard to diabetes-related complications, it may cause an increase in blood glucose levels and neuropathy risk.
\end{abstract}

Keywords: Asthma, Diabetes mellitus, Inhaled corticosteroids

DOI: $10.7176 / \mathrm{JHMN} / 79-08$

Publication date:August $31^{\text {st }} 2020$

\section{Introduction}

Asthma is an inflammatory lung disease affecting $2-17 \%$ of the population and the main type of medication used for the treatment of asthma is inhaled corticosteroids (ICS). ICSs are mainly used for their anti-inflammatory quality. However, disruption of the blood glucose regulation, cataract, glaucoma, osteoporosis and adrenal insufficiency may be experienced when these medications are used in high doses and for a long-term (Heffler at al., 2018).

Diabetes mellitus (DM) is a chronic metabolism disease requiring continuous medical care, where the organism is unable to utilize the carbohydrates, fast and proteins due to the defects in insulin effect or lack of insulin (Al Efraij at al., 2019). To decrease the acute complication risk of the disease and to avoid the chronic sequels requiring costly treatments, the healthcare professionals and the patients should be educated on the matter continuously.

Diabetes mellitus and asthma are two of the most common chronic diseases in the society. The management of both diseases is critical for public health. The effect of oral corticosteroids used in asthma attacks on blood glucose levels are well-known (Sullivan at al., 2018). However, data concerning the management of diabetes in patients using ICS are controversial (Bloom at al., 2019). Although there are studies stating that the use of inhaled corticosteroids is safer than oral or other systemic uses, there are also publications asserting that they may disrupt the diabetes regulation if taken at high doses (Suissa at al., 2010). In this study, it is aimed to investigate the impact of ICSs on the management of diabetes in diabetic patients diagnosed with asthma.

\section{Methods}

This is a retrospective case control study. Patients diagnosed with DM and DM patients diagnosed with asthma were enrolled in the study in two separate sites from January 1, 2018 to January 1, 2020. Patients with both DM and asthma diagnoses were regarded as the study group and patients diagnosed with DM but not asthma were regarded as the control group. The diagnosis of the asthma patients enrolled in the study were based on the guidelines of Global Initiative for Asthma (GINA) (13). Inhaled medications were regulated by a pulmonologist based on GINA. According to these guidelines, patients with respiratory symptoms such as wheezing, shortness of breath, coughing and chest tightness and patients with varying air flow restriction with spirometry were diagnosed with asthma. Patients from both groups were scanned with the automation system of the hospital: the laboratory tests performed at times they have not had a diabetic ketoacidosis attack for DM and they have not had an asthma attack for asthma were recorded in the data form. Inpatients with asthma onset of inflammation, 
progressing shortness of breath, cough, wheezing and chest tightness were defined as the decrease in respiratory functions and requiring systemic steroids for clinical improvement. Diabetic ketoacidosis was defined as the presence of plasma glucose $>250 \mathrm{mg} / \mathrm{dl}$, arterial $\mathrm{pH}<7.30$, serum bicarbonate $<15 \mathrm{mEq} / \mathrm{l}$ and moderate/severe ketonuria and ketonemia. The laboratory tests performed for the study, glycated haemoglobin (HbAlc), fasting blood glucose levels, complete urine tests and creatinine levels were recorded to the form.

Additionally, the diabetes-related complications and number and type of medication used for DM from the patient files were noted on the data from.

All patients diagnosed with DM, who are over 18 were enrolled in the study. Patients using systemic steroids for any reason, patients diagnosed with DM, who do not take medication regularly, patients with diabetic ketoacidosis and patients, who had an asthma episode requiring the use of oral steroids in the past year were excluded from the study. The diabetic complications of the patients were noted from the file system, based on the remarks of the relevant physician following-up the patient.

Local ethics committee approval and academic work permit were obtained before starting the study.

\section{Statistical analysis}

Statistical analysis of the data was carried out using SPSS 23.0 (IBM Corp. Released 2015. IBM SPSS Statistics for Windows, Version 23.0. Armonk, NY: IBM Corp.) statistics software. Definitive statistics such as frequency distribution, average and standard deviation were used to define the samples. In cases, where parametric test assumptions were provided, the difference of both group averages were examined using "Student $t$ test". In cases, where parametric test assumptions were not provided, the non-parametric alternatives of these tests, "MannWhitney U" and "Kruskall Wallis" tests were used. Categorical data were reviewed with "chi-square significance test". Significance level was determined as $\mathrm{p}<0.05$.

\section{Results}

A total of 276 patients; $88(31.9 \%)$ male and $188(68.1 \%)$ male, randomly selected, were enrolled in the study with

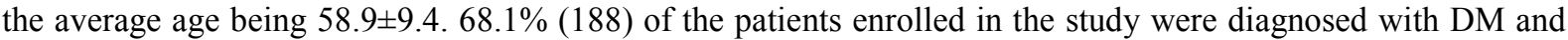
$31.9 \%$ (88) were DM patients diagnosed with asthma. The sex and age distribution of both groups were similar (Table 1). The main characteristics of the patients are shown in Table 1, diabetic complications and medications used are listed in Table 2. Among patients diagnosed with asthma, 6.8\% (6) take low doses and 93.1\% (82) take medium doses, there were not any patient using high dose ICS. 6 of the patients enrolled in the study only used ICS, 36 of the patients used ICS+long-acting beta 2 agonists, and the remaining 46 patients used ICS+long-acting beta2 agonists+montelukast.

Table 1: Main characteristics of patients

\begin{tabular}{|l|c|c|c|}
\hline \multicolumn{1}{|c|}{ Parameter } & Median & Standard deviation & Distribution range \\
\hline Age (years) & 58.9 & 9.6 & $21-28$ \\
\hline Hemogram $(\mathrm{mg} / \mathrm{dl})$ & 13.0 & 1.5 & $8.09-17.00$ \\
\hline HbA1c & 6.5 & 0.9 & $5.00-10.20$ \\
\hline Glucose & 121.5 & 36.9 & $78-297$ \\
\hline Creatinine & 0.78 & 0.20 & $0.43-1.73$ \\
\hline
\end{tabular}

For diabetic patients with asthma, asthma was diagnosed 12.5 \pm 9.6 (1-40) years ago, DM was diagnosed $8.2 \pm 5.2$ (1-23) years ago on average and for patients only diagnosed with DM, the DM was diagnosed 8.3 \pm 7.5 (130) years ago. The most common comorbidities in DM patients diagnosed with asthma were $70.4 \%$ (62) hypertension and $30.0 \%$ (30) hypothyroid, and in patients only diagnosed with diabetes, 58.5\% (110) hypertension and 43.1 (38) hypothyroid.

Table 2: Diabetic complications and medication in all cases

\begin{tabular}{|l|cc|}
\hline Complication & $\mathbf{n}$ & $\mathbf{( \% )}$ \\
\hline Proteinuria & 17 & $(12.3 \%)$ \\
\hline Glycosuria & 3 & $(2.2 \%)$ \\
\hline Nephropathy & 20 & $(14.5 \%)$ \\
\hline Neuropathy & 15 & $(10.9 \%)$ \\
\hline Retinopathy & 5 & $(3.6 \%)$ \\
\hline Medication & $\mathbf{n}$ & $\mathbf{( \% )}$ \\
\hline Insulin & 24 & $(17.4 \%)$ \\
\hline Oral anti-diabetic & 114 & $(82.6 \%)$ \\
\hline
\end{tabular}

The medications most commonly used apart from asthma and diabetes medications were the same in both groups; anti-hypertensives (from most commonly used to the least commonly used; Angiotensin converting enzyme inhibitors, Angiotensin II receptor blockers, Calcium channel blockers, Beta blockers, Diuretics (lowdose)), anti-thyroid medication and proton pump inhibitor. 
The most commonly observed diseases in the family histories of diabetes patients with asthma were $29.5 \%$ (26) DM, 15.9\% (14) asthma and coronary artery disease (each) and $11.3 \%$ (10) hypertension and malignancy (each), and the most commonly observed diseases in the family histories of DM patients not diagnosed with asthma were 54.2\% (102) DM, 29.7\% (56) hypertension, 19.1\% (36) coronary artery disease and hypertension (each). The comparison between DM patients not diagnosed with asthma and the DM patients diagnosed with asthma, blood glucose $(p<0.007)$ levels and incidence of neuropathy $(p<0.002)$ were significantly higher in patients diagnosed with asthma and incidence of nephropathy $(\mathrm{p}<0.024)$ was significantly higher in patients not diagnosed with asthma. In our study, no statistically significant differences were observed between the two groups with regard to age haemoglobin levels and HbA1c levels (Table $3-4$ ).

Table 3: Comparison of continuous variables of both groups

\begin{tabular}{|l|c|c|c|}
\hline & $\begin{array}{c}\text { DM (n:188) } \\
\text { Median+SD }\end{array}$ & $\begin{array}{c}\text { Asthma + DM (n:88) } \\
\text { Median+SD }\end{array}$ & $\mathrm{p}$ \\
\hline Age (years) & $62.3 \pm 9.4$ & $57.6 \pm 9.4$ & 0.950 \\
\hline DM time (year) & $8.3 \pm 7.5$ & $8.2 \pm 5.2$ & 0.936 \\
\hline Haemoglobin $(\mathrm{mg} / \mathrm{dl})$ & $12.6 \pm 1.7$ & $13.2 \pm 1.4$ & 0.268 \\
\hline HbA1c & $6.6 \pm 0.9$ & $6.5 \pm 0.9$ & 0.205 \\
\hline Creatinine $(\mathrm{mg} / \mathrm{dl})$ & $0.78 \pm 0.16$ & $0.77 \pm 0.21$ & 0.570 \\
\hline Glucose $(\mathrm{mg} / \mathrm{dl})$ & $112.7 \pm 20.8$ & $125.7 \pm 41.8$ & 0.007 \\
\hline
\end{tabular}

Table 4: Comparison of categorical data of both groups.

\begin{tabular}{|l|cc|cc|c|}
\hline & \multicolumn{2}{|c|}{$\mathrm{DM}^{*}$} & \multicolumn{2}{c|}{ Ashma+DM } & $\mathrm{p}$ \\
\hline Proteinuria & $\mathrm{n}$ & $(\boldsymbol{\%})$ & $\mathrm{n}$ & $\mathbf{( \% )}$ & \\
\hline Glycosuria & 24 & 12.76 & 10 & 11.36 & 0.815 \\
\hline Nephropathy & 4 & 2.12 & 2 & 2.27 & insufficient data \\
\hline Neuropathy & 24 & 12.76 & 16 & 18.18 & 0.4 \\
\hline Retinopathy & 10 & 5.31 & 20 & 22.72 & 0.002 \\
\hline Insulin & 4 & 2.12 & 6 & 6.81 & insufficient data \\
\hline Female & 56 & 14.89 & 20 & 22.72 & 0.258 \\
\hline
\end{tabular}

*DM: Diabetes mellitus

\section{Discussion}

Incidence of comorbidities in diabetes patients were common, at least half of these patients had three or more comorbid chronic diseases (James at al., 2018). Presence of comorbidities complicate the management of the disease and make the management of the disease costlier for the healthcare systems. Asthma is one of the comorbidities observed in diabetic patients. In earlier studies, the incidence of asthma in patients diagnosed with Type 2 DM was between 0.3-1.5\% and in more recent studies this rate is around 13\% (Guilleminault at al., 2017). In studies performed on children with Type $1 \mathrm{DM}$ diagnosis, the incidence of asthma was determined as $5.7 \%$ (Capucilli at al., 2018).

Inhaled corticosteroids are the main reference point of asthma treatment in all ages. The ICSs have a wide anti-inflammatory activity range. They control the underlying airway inflammation in cases of airway hypersensitivity to allergens. ICS is the only asthma medication that is shown to decrease the rate of mortality and the risk of hospitalization in the long term (Gina, 2016). The majority of the therapeutic benefits are obtained with low doses such as fluticasone $200 \mathrm{mg} /$ day or equivalent, and the incidence of side effects increase in line with the increase in dose (Dales-Yates PT, 2015).

In cases of high dose ICS use, systemic side effects may be observed as a result of absorption from the lungs (Rogala at al., 2019). For this reason, it is important to use the lowest effective dose possible and to decrease the dose even further in patients, whose asthma symptoms are well-managed for several months (Shah at al., 2017). Administration of oral corticosteroids frequently causes deterioration of diabetes, however, there is little data concerning the clinically significant changes in the glucose homeostasis associated with the use of ICS in patients with diabetes. In our study, it was determined that HbA1c levels of patients using ICS do not increase at a statistically significant level, however it was observed that the blood glucose levels were significantly higher in those patients. A prospective, placebo controlled, double-blind study performed by Rogala et al. reached the same conclusion as our study. In the study carried out by Faul et al, the HbAlc levels being normal and the blood glucose levels being higher was assumed to be due to the duration of the study (42 days). It is interesting that in our study, with an average asthma diagnosis duration of 12.5 years, such a similar result was obtained.

In studies carried out in the 1990's, no correlation was found between the use of ICS and DM risk. This is thought to be due to the administration of low-dose ICS at that era (Suissa at al., 2010). In 2007, in an extensive 
study performed on 388,000 patients, it was observed that patients using ICS experienced a disruption of the diabetes regulation and that there was an increase in newly diagnosed diabetes. In this study, the disruption of the diabetes regulation was observed at high ICS doses that are equivalent to a daily $1000 \mathrm{mg}$ dose of Fluticasone or higher. In a study carried out by Choi et al. asthma patients using ICS were compared to asthma patients not using ICS, weight gain, fluid retention, easy bruising, hypertension, DM, epigastric complaints and electrolyte imbalance were more common in the group using ICS.

In addition to these findings, there are studies that show that ICSs do not increase the risk of DM. In a study carried out by Bloom et al. in 2002 and another study carried out by Sonnappa in 2014, it was shown that ICSs do not increase the risk of DM. In our study, it was not examined whether ICS related DM was developed. However, it was observed that diabetic patients with asthma had been living with asthma for an average of 12.5 years and DM for an average of 8.5 years. This finding suggests that in some patients, ICS may have contributed to the diagnosis of diabetes. Although it was concluded that neuropathy, one of the complications of diabetes, was significantly higher in the group using ICS, no statistically significant difference between both groups was observed in regard to the other complications.

Oral corticosteroids are recommended for improving the lung functions and treating hypoxemia, and the short-term treatment of inflammations in order to shorten the recovery time, however, they are not chronically recommended due to their side effects (James at al., 2018). Due to the dose-related increase in blood glucose levels and the increase in the diabetes progression, corticosteroids are not recommended for use in diabetic patients (Coi at al., 2017).

According to the studies carried out $4-60 \%$ of ICSs reach the lungs. The portion of the ICS dose reaching the lungs has the desired pharmacologic effect. A significant portion of the dose reaching the airways may later be absorbed by the systemic circulation with the pulmonary vein structures. (Shah at al., 2017). The remaining portion passes to the systemic circulation through the gastrointestinal system. The medication absorbed by the gastrointestinal channel and comes through without being changed and as not inactivated by the first pass metabolism in the liver, enters the systemic circulation and possible causes additional pulmonary side effects. The corticosteroid entering the systemic circulation disrupts the function of the pancreatic beta cells and may decrease the release of insulin (Volmer at al., 2018). In case of high dose ICS use, the corticosteroid entering the systemic circulation by being absorbed by the liver or the gastrointestinal system may cause disruption in the diabetes regulation.

In diabetes mellitus, the severity of hyperglycaemia is related to microvascular complications. As a general rule, HbA1c (normal individuals having a HbA1c $<6 \%$ ) has a good correlation with the average blood glucose levels measured for the previous $4 \pm 12$ weeks. In the first study indicating that ICSs may disrupt the diabetes regulation, it was observed that high doses $(1000 \mathrm{mg} /$ day $)$ of fluticasone has caused an increase in the level of $\mathrm{HbAlc}$ and glycosuria. However, in our study, although the number of patients enrolled was low, no statistically significant differences were observed between two groups. This may be due to the fact that the patients used lowmedium doses of ICS.

Corticosteroids decrease the level of insulin secreted, increase insulin resistance and stimulate gluconeogenesis in the liver, causing hyperglycaemia (Tashkin at al., 2018). It was shown that corticosteroids inhibit certain steps within the insulin signal network causing insulin resistance. These include increased proteolysis, lipolysis and production of free fatty acids, and this contributes to insulin resistance. Corticosteroids may also increase direct hepatic gluconeogenesis and this causes hyperglycaemia (De Micheli A, 2016). However, in our study, no statistically significant differences were detected with regard to the frequency of insulin use between diabetic patients diagnosed with asthma and diabetic patients not diagnosed with asthma. This may be due to the fact that the majority of the patients in our study used ICSs in low-medium doses and none of them used ICSs in high doses.

In 2018 James et al. reported that despite the fact that the negative effects of corticosteroids (both oral and inhaled) on diabetes are significant in only high doses, the blood glucose levels of this patient population should be followed up closely. They also reported that in this group of patients (patients using high doses of corticosteroids), the treatment effectiveness should be reviewed 4-8 weeks after the start of inhaler treatment and regular use of high-dose ICSs should be avoided in patients who also have diabetes.

The most important restrictions of this study are that the diabetic complications were checked from the patient files and the number of patients, especially the ones using ICS in high doses was low.

\section{Conclusion}

In conclusion, the use of low-medium dose ICSs in diabetes mellitus patients diagnosed with asthma does not have an impact on $\mathrm{HbA1c}$ levels but with regard to diabetes-related complications, it may cause an increase in blood glucose levels and neuropathy risk. It would be beneficial if this study is carried out in a better-planned manner and with sufficient number of patients using low-medium-high doses of ICS. 


\section{References}

Al Efraij K, Johnson KM, Wiebe D, Sadatsafavi M, FitzGerald JM. A systematic review of the adverse events and economic impact associated with oral corticosteroids in asthma. J Asthma. 2019;56(12):1334-1346. doi:10.1080/02770903.2018.1539100

Bloom CI, Douglas I, Olney J, D'Ancona G, Smeeth L, Quint JK. Cost saving of switching to equivalent inhalers and its effect on health outcomes. Thorax. 2019;74(11):1078-1086. doi:10.1136/thoraxjnl-2018-212957.

Capucilli P, Cianferoni A, Grundmeier RW, Spergel JM. Comparison of comorbid diagnoses in children with and without eosinophilic esophagitis in a large population. Ann Allergy Asthma Immunol. 2018;121(6):711-716. doi: 10.1016/j.anai.2018.08.022.

Choi IS, Sim DW, Kim SH, Wui JW. Adrenal insufficiency associated with long-term use of inhaled steroid in asthma. Ann Allergy Asthma Immunol. 2017;118(1):66-72. e1. doi: 10.1016/j.anai.2016.10.002.

Daley-Yates PT. Inhaled corticosteroids: potency, dose equivalence and therapeutic index. Br J Clin Pharmacol. 2015;80(3):372-380. doi:10.1111/bcp.12637.

De Micheli A. Il diabete steroideo: inquadramento e gestione [Corticosteroid induced diabetes mellitus: diagnosis and management]. G Ital Nefrol. 2016;33(S68): gin/33. S68.7.

GINA. 2016-Pocket Guide for Asthma Management and Prevention, GINA Foundation, 2016. Available at: nasthma.org/wp-con-tent/uploads/2016/05/WMS-GINA2016-main-Pocket-Guide.pdf.

Guilleminault L, Williams EJ, Scott HA, Berthon BS, Jensen M, Wood LG. Diet and Asthma: Is It Time to Adapt Our Message? Nutrients. 2017;9(11):1227. Published 2017 Nov 8. doi:10.3390/nu9111227.

Heffler E, Madeira LNG, Ferrando M, et al. Inhaled Corticosteroids Safety and Adverse Effects in Patients with Asthma. J Allergy Clin Immunol Pract. 2018;6(3):776-781. doi: 10.1016/j.jaip.2018.01.025

James BD, Jones AV, Trethewey RE, Evans RA. Obesity and metabolic syndrome in COPD: Is exercise the answer? Chron Respir Dis. 2018;15(2):173-181. doi:10.1177/1479972317736294.

Kamada AK, Szefler SJ, Martin RJ, et al. Issues in the use of inhaled glucocorticoids. The Asthma Clinical Research Network. Am J Respir Crit Care Med. 1996;153(6 Pt 1):1739-1748. doi:10.1164/ajrccm.153.6.8665030.

Rogala B, Bożek A, Gluck J. Is there a relationship between asthma and diabetes? [published online ahead of print, 2019 Aug 20]. J Asthma. 2019;1-7. doi:10.1080/02770903.2019.1652642.

Shah SS, Ohlsson A, Halliday HL, Shah VS. Inhaled versus systemic corticosteroids for the treatment of bronchopulmonary dysplasia in ventilated very low birth weight preterm infants. Cochrane Database Syst Rev. 2017;10(10):CD002057. Published 2017 Oct 16. doi: 10.1002/14651858.CD002057.pub4.

Sonnappa S, Martin R, Israel E, et al. Risk of pneumonia in obstructive lung disease: A real-life study comparing extra-fine and fine-particle inhaled corticosteroids. PLoS One. 2017;12(6): e0178112. Published 2017 Jun 15. doi: 10.1371/journal.pone.0178112.

Suissa S, Kezouh A, Ernst P. Inhaled Corticosteroids and the Risks of Diabetes Onset and Progression. Am J Med. 2010; 123:1001-1006.

Sullivan PW, Ghushchyan VH, Globe G, Schatz M, Oral Corticos-teroid Exposure and Adverse Effects in Asthma, Journal of Allergy and Clinical Immunology 2018; 141(1):110-116.

Tashkin DP, Wechsler ME. Role of eosinophils in airway inflammation of chronic obstructive pulmonary disease. Int J Chron Obstruct Pulmon Dis. 2018; 13:335-349. Published 2018 Jan 17. doi:10.2147/COPD.S152291.

Volmer T, Effenberger T, Trautner C, Buhl R. Consequences of long-term oral corticosteroid therapy and its sideeffects in severe asthma in adults: a focused review of the impact data in the literature. Eur Respir J. 2018;52(4):1800703. Published 2018 Oct 25. doi:10.1183/13993003.00703-2018. 